\title{
Genre, diffusion et adoption des technologies de gestion durable des terres dans les petites exploitations familiales des pays en voie de développement : une revue
}

\author{
Sourou T. Christian ADJIBA ${ }^{1 *}$, Ygue Patrice ADEGBOLA ${ }^{3}$ et Jacob A. YABI ${ }^{1,2}$ \\ ${ }^{1}$ Laboratoire d'Analyse et de Recherches sur les Dynamiques Economiques et Sociales (LARDES), \\ Département d'Economie et de Sociologie Rurales, Faculté d'Agronomie, Université de Parakou, BP 123 \\ Parakou, République du Bénin. \\ ${ }^{2}$ Faculté d'agronomie de l'Université de Parakou (FA/UP), Parakou, République du Bénin. \\ ${ }^{3}$ Institut National de Recherche Agronomique du Bénin (INRAB). \\ *Auteur correspondant ; E-mail : addadjiba@gmail.com
}

Received: 25-03-2021

Accepted: 15-10-2021

Published: 30-10-2021

\section{RESUME}

La gestion durable des terres (GDT) est devenue un moyen pour combattre l'insécurité alimentaire et fournir un revenu adéquat aux producteurs dans les Pays En Voie de Développement (PEVD) face à la baisse de la fertilité des sols. Cet article avait pour but de synthétiser 145 documents sélectionnés dans la base de données Scopus menés sur les technologies de GDT pour ressortir les facteurs clés qui motivent leur adoption et les approches de diffusion appréciées pour leur mise à l'échelle. Les déterminants ont été regroupés en 4 sousgroupes. Le premier groupe était constitué des facteurs psycho-sociodémographiques. Le deuxième groupe était constitué des facteurs économiques. Le troisième groupe était constitué des facteurs institutionnels et le quatrième niveau concernerait les facteurs biophysiques. Cette revue et synthèse attire l'attention sur le fait de rendre palpable la participation des producteurs en les plaçant au cœur du processus d'adoption. Améliorer les taux d'adoption faible constaté passe donc par une batterie de mesures de politiques agricoles dans ces PEVD. Il faudra proposer des technologies types adaptées aux profils des agriculteurs et à l'environnement de leurs parcelles tout en travaillant sur l'amélioration de leur condition économique en passant par la fourniture de services institutionnels adéquats. Une attention particulière doit être apportée à la gente féminine qui est souvent laissée pour compte.

(C) 2021 International Formulae Group. All rights reserved.

Mots clés : GDT, Mise à l'échelle, Facteurs, Adoption, Pays en voie de développement, Genre.

\section{Gender, diffusion and adoption of sustainable land management technologies in small family farms in developing countries: a review}

\section{ABTRACT}

Sustainable land management (SLM) has become a means to combat food insecurity and provide adequate income to smallholders in developing countries (DC). This article synthesizes 145 papers selected from the SCOPUS database conducted on SLM technologies to highlight the key 
factors that motivate their adoption and the dissemination approaches appreciated for their scaling up. The determinants were grouped into 4 subgroups. The first group is made up of psycho-sociodemographic factors. The second group is made up of economic factors. The third group consisted of institutional factors and the fourth level concerned biophysical factors. This review and synthesis draws attention to making the participation of smallholders tangible by placing them at the heart of the adoption process. Improving the observed low adoption rates therefore requires a battery of agricultural policy measures in these DC. It will be necessary to offer standard technologies adapted to the profiles of farmers and the environment of their plots while working on improving their economic condition through the provision of adequate institutional service. Particular attention must be paid to women who are often overlooked.

(c) 2021 International Formulae Group. All rights reserved.

Keywords: SLM, Scaling-up, Factors, Adoption, Developing countries, Gender.

\section{INTRODUCTION}

La pression démographique engendre des conséquences néfastes sur l'environnement à travers la déforestation. Ceci conduit les producteurs à la recherche de nouvelle friche pour produire. Cela a pour corolaire la dégradation des terres qui est une sérieuse menace pour la production dans les pays en voie de développement. Elle se présente généralement sous forme d'érosion des sols(Diao and Sarpong, 2011; Dessie et al., 2012). La gestion durable des terres (GDT) est un moyen important pour accroitre la productivité et améliorer la sécurité alimentaire (Teshome et al, 2014). Elle est aussi un moyen d'adaptation au changement climatique par son action de séquestration du carbone organique du sol (Salé et al, 2014; Asfaw et al., 2016; Cholo et al., 2018; Lokonon and Mbaye, 2018, Adébiyi et al, 2019). Une adoption des bonnes pratiques de GDT pourrait favoriser une meilleure gestion des ressources naturelles et asseoir les fondements d'un développement économique et social durable (Kohio et al, 2017). La GDT est donc une alternative pour remédier aux problèmes du sol. Elle englobe plusieurs gammes de technologies comme l'agroforesterie, l'association rotation et les technologies de lutte antiérosive mis en lumière dans les travaux de Yabi et al, (2018). Dans cette étude, la GDT est définie comme un ensemble complet de pratiques de gestion des terres, avec le potentiel d'apporter des différences significatives et durables dans le futur proche et à long terme en termes de réduction de la dégradation des sols et d'amélioration de leur productivité (Liniger et al. 2011).

Ce concept a mobilisé la littérature ces 30 dernières années (Teklewold and Kohlin, 2011; Okpara et al., 2019; Carriazo et al., 2020). Cependant leur adoption reste très contrasté (Adégbola et al., 2010; Vidogbéna et al., 2016). Pour expliquer cet état de chose, les économistes se positionnent sur le déterminisme de l'adoption tandis que les sociologues ont investigué les approches de GDT et les stratégies de mise à l'échelle (Chinseu et al., 2019). La compréhension de ces différents paramètres s'avère crucial pour accroitre le taux d'adoption des technologies de GDT et de permettre la formulation d'option de politiques agricoles pour améliorer le bienêtre des populations rurales. Plusieurs travaux de synthèse ont été élaborés sur les facteurs déterminants l'adoption (Shiferaw et al., 2009; Tey et al., 2017). Très peu se sont essayés à synthétiser les approches de diffusion des GDT.

C'est pour combler cette insuffisance que la présente étude se propose de synthétiser les différentes publications sur les technologies de GDT concernant les approches et les facteurs déterminants l'adoption. L'objectif principal de cet article était donc de faire une revue et synthèse sur les parutions en technologies de GDT en mettant particulièrement l'accent sur les différentes approches de mise à l'échelle. Après avoir expliqué la méthodologie ayant conduit à la 
sélection des articles et les méthodes d'analyse, la deuxième section s'est focalisée sur les approches de diffusion des GDT, la troisième section s'est concentrée sur les différents facteurs qui gouvernent l'adoption des technologies de GDT et cette revue se termine par une conclusion.

\section{MOTEUR DE RECHERCHE ET DOCUMENTS CONSULTÉS}

Pour faire cette revue et synthèse, le moteur de recherche en ligne AGORA a été utilisé. Les documents publiés dans la base SCOPUS ont été retenus. Les documents sélectionnés couvraient tous les articles et revues de littérature, publiés de 1983 à 2019 sur les pratiques de GDT concernant les pays en voie de développement d'Afrique, d'Asie et d'Amérique latine dans cette large base de données. Les articles ont aussi tenu compte de l'homogénéité des zones qui ont été exclues de cette revue et synthèse, tous les articles venant d'Amérique du Nord, d'Europe et d'Océanie (Nouvelle-Zélande et Australie particulièrement). En d'autres termes les zones géographiques à climat tempéré et regroupant les pays développés ont été exclus.

Deux expressions ont été utilisé premièrement «sustainable land management » et «adoption » pour extraire les documents compatibles avec l'objectif visé dans cette revue de littérature et synthèse. La période prise en compte va du début des publications d'articles traitant des aspects relatifs à la GDT dans Scopus. A ce premier stade, 727 articles ont été retenus. Après ce premier résultat obtenu, des articles trouvés par domaines ont été triés et analysés. Ainsi les domaines «Economie, Econométrie et Finance », «Sciences sociales », «Agriculture et Sciences biologiques» et « Sciences environnementales » ont été considéré. De ce $2^{\mathrm{e}}$ tamisage, la pertinence des articles avec l'objectif du présent article a été analysée. Ainsi, certains articles qui ont échappé au premier tamisage ont été retirés. Ce processus a permis de retenir 145 publications (Figure 1).

\section{MISE A L'ECHELLE DES TECHNOLOGIES DE GDT}

Une approche de GDT est tout arsenal mis en place pour favoriser l'adoption d'une technologie de GDT. Selon une récente étude conduite en Ethiopie, les approches de GDT englobent les moyens de soutenir et de permettre aux agriculteurs de mettre en œuvre, d'adopter et d'adapter la GDT sur les terres agricoles (Abi et al., 2018). Le taux d'adoption ne dépend pas seulement des facteurs mais aussi des approches utilisées pour leur diffusion. Malheureusement une faible proportion, $14 \%$ des articles considérés dans cette synthèse ont abordé la mise à l'échelle des technologies de GDT à travers diverses approches (Figure 2). Seulement les publications que nous avons qualifiées de mixe et celles qualitatives ont abordé les approches de mise à l'échelle respectivement à $13 \%$ et $39 \%$ de leur proportion. Aucune recherche quantitative n'a abordé les approches de GDT. Cette différence est significative au seuil de $1 \%$ $($ Pearson chi2 $(2)=13.7157 \operatorname{Pr}=0.001)$.

Selon l'évolution temporelle des approches, trois périodes peuvent globalement être distinguées. Shiferaw et al., (2009) dans une étude synthèse en Ethiopie, a spécifié 3 types d'approches pour la Conservation des Eaux et Sol (CES) selon l'évolution historique au cours du temps. Il s'agit des interventions descendantes (Top Down) qui ont duré jusqu'aux années 1980, les approches populistes ou paysannes et les approches néolibérales. L'approche descendante était basée sur des méthodes structurelles ou on observe une absence totale de la capacité d'innovation des agriculteurs. Cela se résume en un transfert de technologie (TOT). Quant à l'approche populiste, elle plaçait l'agriculteur comme élément central de la conception des programmes et la mise en œuvre d'activités. Très tôt cette approche a été dépassée car il était difficile de réellement prendre de décision dans ce schéma. Une $3^{\text {e }}$ approche est alors apparue. Il s'agit de l'approche néolibérale qui a reconnu les rôles appropriés pour l'innovation paysanne mais mettait au centre le 
rôle critique des marchés, des politiques et des institutions pour stimuler et inciter les paysans à innover, adopter et adapter les options appropriées. C'est cet ensemble d'approche de CES qui a évolué pour donner le concept de GDT (Shiferaw et al., 2009). Certaines études ont conclu qu'une approche descendante n'aurait pas d'impact durable sur la fertilité des sols et la sécurité alimentaire ( Tougiani et al., 2009; Abi et al., 2018). Les producteurs rejetaient ou ne participaient aux interventions qui étaient sous la menace des actions coercitives exercées parfois par l'armée. Cela a conduit au développement des approches plus flexible (Chinseu et al., 2019). Des résultats d'étude corroboraient cette constatation en mentionnant les approches participatives comme un moyen pouvant permettre une implémentation réussie des technologies de GDT (Amede et al., 2007; Bertin et al., 2014; Cordingley et al., 2015). Les approches participatives de la gestion des terres impliquant les parties prenantes ont contribué grandement à une productivité durable et à la conservation des terres (Stringer et al.,2014; Cordingley et al., 2015). Les chercheurs et scientifiques en amont conçoivent des innovations technologiques (ici technologies de GDT) à l'endroit des producteurs. Malgré que ces technologies visent l'amélioration des conditions de vie de ces derniers, un faible taux d'adoption est observé (Drechsel et al., 1996; Bertin et al., 2014; Clay et al., 2014; Cordingley et al., 2015; Emerton and Snyder, 2018). L'une des causes de cet état de chose est lié à l'approche utilisée depuis le design de la technologie jusqu'à sa mise en œuvre. Souvent, la faible utilisation des mesures de GDT est due à un échec depuis la conception jusqu'à la mise en œuvre d'approches de GDT permettant de reconnaître pleinement les intérêts des gestionnaires de terres et la dimension socioéconomique (Fleskens et al., 2014). Plusieurs études ont révélé que les projets de GDT échouent parce qu'ils n'ont pas prise en compte les réalités des populations autochtones depuis la planification jusqu'à leur mise en œuvre. Des exemples de succès ont été signalés en
Ethiopie et en Tanzanie lorsque la participation des producteurs a été effective, les comportements ont été modifié en faveur de l'adoption de technologie de GDT (Amede et al., 2007; Kwayu et al., 2014; Blake et al., 2018). La participation des producteurs au design des projets sur les technologies de GDT renforcerait donc le taux d'adoption des technologies (Chinseu et al., 2019). Une leçon apprise de cette dernière étude est qu'au Malawi, cela s'est traduit à travers une réponse adéquate au réel besoin des destinataires finaux, mettant plus l'accent sur la résilience de l'Agriculture de Conservation (AC) que sur ces bénéfices économiques et sur les connaissances et les expériences paysannes dans le développement de paquet technologiques de l'AC plus compatibles, plus flexibles et à un prix d'intrant réduit. Cette participation vise aussi à valoriser les savoirs et compétences endogènes. La valorisation des connaissances endogènes est très importante dans la mise à en œuvre des pratiques de GDT ( Zurayk et al., 2001; Siedenburg, 2005; Homann et al., 2008; Dessie et al., 2012; Singh et al., 2014; Magni, 2017). La valorisation de ce type de connaissance encouragerait également l'adoption de pratiques de GDT en établissant un partenariat et un dialogue entre les parties prenantes (Zurayk et al., 2001). Il serait capital d'associer les acteurs à la base depuis le design des pratiques de GDT (Jones, 1997; Blake et al., 2018). Mais une contrainte de l'approche participative serait que les connaissances autochtones utiles et intéressantes sont souvent rares (Cools et al., 2003).

Pour les initiatives de GDT en cours et à venir, l'amélioration du taux l'adoption des technologies de GDT passe par les étapes suivantes : (1) la conception de technologie de GDT plus adaptée à l'utilisateur final et donc plus susceptible d'entraîner des améliorations des moyens de subsistance; (2) la résolution des contraintes institutionnelles et (3) l'identification des situations dans lesquelles des mécanismes d'incitation durables à long terme sont nécessaires pour permettre aux utilisateurs des terres d'adopter la GDT 
(Cordingley et al., 2015). Aussi, les approches de GDT ne doivent pas seulement placer la santé du sol en $1^{\text {er }}$ et accorder une faible importance aux améliorations des moyens de subsistance (Silas, 2014; Cordingley et al., 2015). Lorsque l'adoption de la technologie de GDT dans certains cas est motivée par d'importants fonds de donateurs, cela met en péril la durabilité à long terme de la technologie de GDT employée. Pour remédier à cela, il est nécessaire d'encourager l'utilisation de mécanismes basés sur des marchés incitatifs (Branca et al., 2013; Montagnini and Finney, 2011; Silas, 2014). Les mécanismes possibles proposés peuvent être regroupés en systèmes de paiement publics, en transactions ouvertes régies par la réglementation, en transactions privées autoorganisées et en éco-labing de produits et services. Des études futures pourraient abonder dans ce sens.

\section{Facteurs déterminants l'adoption ou l'investissement dans les pratiques de GDT}

L'adoption d'une innovation est vecteur d'un ensemble de facteurs tant visibles qu'invisibles, endogène, qu'exogène. Cette revue de littérature fournit un large corpus de connaissance sur cet aspect. Souvent, les mêmes facteurs qui entraînent la dégradation des terres empêchent aussi l'adoption de ces pratiques de GDT (Shiferaw et al., 2009). La pauvreté entraînant un manque de capital pour l'investissement, un régime foncier précaire, des infrastructures et des services de vulgarisation limités, des prix du marché volatils ou peu fiables pour les produits et intrants agricoles, le manque d'accès au crédit et à la main-d'œuvre, sont autant de facteurs sociaux qui peuvent entraver l'adoption de la GDT. La décision d'adopter une technologie de GDT dépend alors d'un large éventail de facteur qui peuvent être spécifique à une zone, au ménage ou aux caractéristiques de la parcelle (Teklewold and Kohlin, 2011).

Cette synthèse fournit un riche éventail de connaissances, permettant de mieux cerner les facteurs permettant de mieux comprendre le mécanisme d'adoption des technologies de GDT. Au regard de ces facteurs, une typologie en 4 groupes de facteurs a été proposée à savoir les facteurs psycho-sociodémographiques, les facteurs économiques, les facteurs institutionnels et les facteurs biophysiques.

\section{Facteurs psycho-sociodémographiques}

Les facteurs psychosociodémographiques concernent l'âge, le sexe, l'éducation, l'accès à l'information, l'expérience en agriculture, la formation technique, la densité de la population, le ratio homme/terre, l'appartenance à une organisation, l'attitude, l'aversion au risque. Parmi les articles analysés, 17,92\% (19 articles) ont montré l'importance de l'âge dans la décision d'adoption des technologies de GDT (Figure 3). L'âge s'avère un facteur important dans le processus d'adoption de certaines technologies de GDT (Shiferaw, 1998; Teklewold and Kohlin, 2011; Wossen et al., 2013; Wollni and Andersson, 2014; Ndiritu et al., 2014; Kabubo-Mariara, 2015; Ashoori et al., 2016;Asfaw et al., 2016; Obayelu et al., 2016; Nigussie et al., 2017; Etongo et al., 2018; Gao et al., 2018; Abi et al., 2018; Nkomoki et al., 2018). Mais son rôle est assez ambigu. L'âge a influencé positivement l'adoption du zaï (Etongo et al., 2018) et aussi le fumier de ferme, les diguettes de pierres traditionnelles, le Fanya Juu et de l'AC (Kabubo-Mariara, 2015; Abi et al., 2018).Cela signifie que les producteurs âgés maitrisant mieux ces technologies, cela faciliterait le processus d'adoption à leur niveau. L'autre explication pourrait être que les producteurs âgés sont plus enclins à conserver les technologies traditionnelles de gestion de la fertilité de leur sol. Une autre raison serait l'horizon de planification plus long pour les jeunes par rapport aux plus âgés pour bénéficier des avantages de la technologie. Par contre, l'âge a révélé une influence négative sur l'adoption des technologies de CS. Ceci traduirait le fait que les producteurs jeunes adoptent ces technologies de GDT que les plus âgés (Teklewold and Kohlin, 2011; Ashoori et al., 2016). Cela est induit par l'intensité de la main d'œuvre en faveur des jeunes, ce qui constitue un avantage sur les plus âgés. 
Concernant le sexe, $13,21 \%$ des articles, le suggère comme facteur clé dans la décision d'adoption soit 14 articles sur les 106 (Figure 3). Des travaux empiriques ont rapportés l'importance du sexe dans l'adoption de certaines technologies de GDT (Shiferaw, 1998; Pender and Gebremedhin, 2007; Wossen et al., 2013; Ndiritu et al., 2014; Asfaw et al., 2016; Ashoori et al., 2016; Obayelu et al., 2016; Nigussie et al., 2017; Tey et al., 2017; Nkomoki et al., 2018; Zeweld et al., 2018; Abi et al., 2018; Issahaku and Abdul-Rahaman, 2019; Teshager Abeje et al., 2019). Les conclusions de Abi et al., (2018) ont rapporté que le sexe a influencé négativement l'épandage du fumier et positivement l'engrais inorganique. Ceci est dû au fait que les femmes souvent dépourvues de certains moyens de subsistance n'arrivent pas à investir dans l'amélioration de leur terre ou parce qu'elles ne disposent pas suffisamment d'énergie pour certaines technologies consommatrices en facteur travail comme les technologies de CES. Par contre, Obayelu et al., (2016) ont trouvé que le sexe influençait positivement l'adoption d'engrais inorganique et la gestion de l'eau au Nigéria. Toujours dans ce sens, le sexe a également influencé positivement l'adoption du compost et de l'agroforesterie par contre négativement la rotation de culture (Zeweld et al., 2018). Ceci traduit que les femmes ont adopté l'épandage du fumier contrairement à la fertilisation inorganique priorisée par les hommes et à la rotation de culture parce que les femmes disposent de peu de terre pour pratiquer des rotations. Ces dernières conclusions sont étayées par Tey et al., (2017) qui ont montré que les producteurs de sexe masculin influencent positivement l'adoption de technologies de gestion durable des terres. Les conclusions montrent cependant un effet contrasté du sexe selon les technologies de GDT.

L'éducation a été rapportée comme un facteur pouvant impacter l'adoption de technologies de GDT. De l'ensemble des articles considérés ici, 20\% $\quad(\mathrm{n}=21) \quad$ ont mentionné l'éducation comme facteur clé (Figure 3). Des travaux empiriques ont relaté cette évidence (Paudel and Thapa, 2004; Pender and Gebremedhin, 2007; Ahaneku,
2010; Wossen et al., 2013; Teklewold et al., 2013; Ndiritu et al., 2014; Kabubo-Mariara, 2015; Asfaw et al., 2016; Ashoori et al., 2016; Nigussie et al., 2017; Tey et al., 2017; Abi et al., 2018; Gao et al., 2018; Etongo et al., 2018; Lokonon and Mbaye 2018; Nkomoki et al., 2018; Zeweld et al., 2018, Issahaku and AbdulRahaman, 2019; Teshager Abeje et al., 2019). L'éducation apparait comme un facteur important qui permet d'avoir une capacité d'analyse et de prise de décision. Elle permet de mieux percevoir par l'exemple l'érosion des sols, de renforcer les capacités d'analyses des coûts et de jauger s'il faut aller vers un emploi extra agricole ou non (Adimassu et al., 2016). L'effet de l'éducation reste cependant ambigu sur l'adoption de technologies de GDT. Des résultats empiriques ont montré que l'éducation a affecté positivement certaines technologies de GDT à l'instar de l'épandage du fumier (Abi et al., 2018), de l'adoption des semences améliorées (Teklewold et al., 2013) et de l'adoption de nouvelle mesures de gestion des ressources naturelles (Wossen et al., 2013). Par contre, elle a affecté négativement l'adoption de l'engrais organique, des diguettes de sols revêtues de pierres et du Fanya Juu (Abi et al., 2018). L'effet de l'éducation est souvent vu comme une variable qualitative binaire qui mesure si oui ou non le producteur a reçu une forme d'éducation mais une étude avait plutôt mis la précision sur son aspect quantitatif à travers le nombre d'année d'écolage du chef ménage comme facteur d'adoption de technologies de GDT (Paudel and Thapa, 2004).

Par contre 10\% (11 articles) des articles ont évoqué l'accès à l'information comme facteur déterminant l'adoption des technologies de GDT (Figure 3). L'accès à l'information est aussi crucial dans l'adoption des technologies de GDT (Caviglia-Harris, 2003; Clay et al., 2014; Chalak et al., 2017; Cholo et al., 2018; Issahaku and AbdulRahaman, 2019; Razzaghi Borkhani and Mohammadi, 2019). Les résultats des travaux de Cholo et al., (2018) ont révélé que l'accès à l'information favoriserait l'adoption du fumier, de la plantation de l'Enset, du terrassement et de la plantation d'arbres indigènes. L'accès à l'information est aussi possible par l'éducation 
informelle à savoir l'alphabétisation mentionnée par certains travaux empiriques. Par exemple, l'adoption de l'agroforesterie, du compost et de la rotation de culture ont ainsi été influencés par l'alphabétisation (Zeweld et al., 2018). L'accès à l'information permettrait de connaitre d'abord les différentes options de technologies existantes par le producteur et de choisir parmi les options qui sont disponible (Caviglia-Harris, 2003). Le manque de connaissance qui n'est remédiable qu'au travers de l'information découragerait l'adoption des technologies d'intensification durable (Rahman et al., 2017)

La taille de ménage est aussi mentionnée par 21 études comme facteur impactant les décisions dans l'adoption des technologies de GDT soit $20 \%$ des articles (Figure 3). La taille du ménage affecte l'adoption des technologies de GDT (Shiferaw, 1998; Ajayi et al., 2007; Pender and Gebremedhin, 2007; Wossen et al., 2013; Ndiritu et al., 2014; Asfaw et al., 2016; Ashoori et al., 2016; Etongo et al., 2018; Martey et al., 2019; Teshager Abeje et al., 2019; Zeweld et al., 2018). Son effet demeure controversé car elle a influencé aussi bien positivement que négativement l'adoption des technologies de GDT. Une taille de ménage élevée est un proxy qui exprimerait la dotation en main d'œuvre familiale (Paudel and Thapa, 2004; Tesfaye et al., 2014; Nigussie et al., 2017; Cholo et al., 2018; Zeweldet al., 2018;Etsay et al., 2019) à priori pour un ménage et donc favoriserait l'adoption des technologies consommatrices en main d'œuvre comme les technologies de Conservation des Eaux (CE) (Wossen et al., 2013). Par contre Ashoori et al., (2016) ont trouvé le contraire toujours sur les technologies de Conservation des Sols (CS) par la réaffectation de cette main d'œuvre à d'autres activités compte tenu du coût d'opportunité de cette dernière. Vidogbéna et al., (2016) ont aussi montré qu'une forte demande en main d'œuvre limitait l'adoption des technologies agro-écologiques. En sommes, 5,66\% ( $\mathrm{n}=6)$ des articles ont évoqué la dotation en main d'œuvre familiale comme facteur pouvant influencer l'adoption des technologies de GDT.
L'expérience en agriculture est aussi un autre facteur psycho-sociodémographique. Elle a été mentionnée dans 8,49\% ( $\mathrm{n}=9)$ des articles étudiés (Figure 3). Des études récentes ont mis en exergue l'expérience en agriculture dans l'adoption de technologies de GDT (Chalak et al., 2017; Cholo et al., 2018; Nkomoki et al., 2018; Zeweld et al., 2018; Cortner et al., 2019; Martey et al., 2019). Ce facteur fortement corrélé à l'âge pousse les producteurs âgés à être réfractaire dans l'adoption de certaines technologies qui leur présentent une fenêtre de planification courte. C'est tout à fait le contraire chez les jeunes qui investissent dans les technologies dont le retour sur investissement n'est perceptible que sur le moyen ou le long terme.

La formation technique joue aussi un rôle dans l'acquisition de compétences dans l'adoption de technologies de GDT (Bodnár and De Graaff, 2003; Paudel and Thapa, 2004; Ahaneku, 2010; Tesfaye et al., 2014; Chalak et al., 2017; Zeweld et al., 2018). Ce facteur est abordé par 7\% ( $\mathrm{n}=7)$ des articles retenus pour cette synthèse (Figure 3 ). Les nouvelles technologies demandent un certain type de savoir-faire et les études suscitées ont montré que lorsque les producteurs étaient suffisamment formés, ils ont adopté de nouvelles technologies.

Un autre facteur psychosociodémographique est le capital social abordé par 7\% $(\mathrm{n}=7)$ des articles. Ce facteur joue un rôle crucial dans l'adoption de technologies de GDT (Caviglia-Harris, 2003; Teklewold et al., 2013; Wossen et al., 2013; Ndiritu et al., 2014; Asfaw et al., 2016; Lokonon and Mbaye, 2018; Zeweld et al., 2018; Cortner et al., 2019) et peut être défini comme le capital relationnel et l'appartenance à un groupe. Wossen et al., (2013) ont montré que la taille des réseaux sociaux d'un ménage dans un village affectait positivement l'adoption de technologies de Gestion des Ressources Naturelles (GRN). Par le capital social, les agriculteurs accèdent plus facilement à l'information qui leur permet d'éliminer les incertitudes auxquelles ils sont sujets et cela façonne une perception positive de la nouvelle technologie. L'addition d'un 
ami, d'un membre de la famille et d'un voisin ayant déjà adopté une technologie a accentué aussi l'adoption de nouvelles technologies de GRN (Wossen et al., 2013). Une influence positive du capital social a été mentionnée au Honduras par l'accès à l'information (Wollni and Andersson, 2014). Cette même étude a montré que la conformité sociale a joué un rôle important dans la décision d'adoption des agriculteurs. Les agriculteurs étaient beaucoup plus susceptibles d'adopter par exemple l'agriculture biologique s'ils pensent que leurs voisins approuveraient leur décision. L'affiliation sociale, qui est un fort moyen entrant dans le capital social des peuples endogènes, à une caste ou à des normes sociales préétablies a affecté l'adoption des technologies de GDT (Paudel and Thapa, 2004, Cortner et al., 2019).

L'attitude joue également un rôle crucial dans l'adoption des technologies de GDT et a été abordée par $4 \%(n=4)$ des articles (Figue 3). Les agriculteurs qui ont une attitude très positive (la facilité perçue, à l'utilité perçue et à la compatibilité perçue) à l'égard de certaines technologies les adoptent (Shiferaw, 1998). C'est ce que rapporte une étude récente conduite en Ethiopie ou les producteurs étaient plus susceptibles d'adopter la rotation avec les légumineuses (Zeweld et al., 2018) contrairement aux systèmes agro-forestiers. La perception sur la fertilité a aussi joué un rôle positive dans l'adoption de technologies de GDT (Ndiritu et al., 2014; Tesfaye et al., 2014; Kabubo-Mariara, 2015).

Les croyances sociétales ou les traditions locales face à certaines technologies peuvent être un frein à leur adoption (Clay et al., 2014; Rahman et al., 2017). Seulement 3\% $(n=3)$ des articles l'ont abordé. Le ratio (terre/homme) d'accès à la terre a déterminé en Ethiopie l'adoption de certaines technologies de GDT (Shiferaw, 1998; Belay and Bewket, 2013). La densité de la population a eu des incidences positives dans l'adoption de technologies de CS dans certains pays (Bodnár and De Graaff, 2003; Pender and Gebremedhin, 2007; Kabubo-Mariara, 2015).

L'aversion au risque ou les attitudes à l'égard des risques (Zeweld et al., 2018) est un facteur qui a affecté la décision des producteurs. Les agriculteurs veulent prendre une décision en fonction de preuves objectives et de caractéristiques spécifiques plutôt que de l'activité en elle-même. L'aversion au choc et stress, a influencé aussi l'adoption de certaines technologies de GDT (Teshager Abeje et al., 2019). Une incertitude pour les producteurs comme évoqué serait le fait de sacrifier un revenu à court terme pour un bénéfice incertain à long terme qui résulterait des décisions d'adoption des technologies de GDT. Cela dénote d'une attitude de prise de risque pour ces agriculteurs. Dans cette revue, certaines études ont fait mention du risque dans l'agriculture (Shiferaw et al., 2009; Teklewold and Kohlin, 2011; Dessalegn et al., 2018; Gao et al., 2018; Zeweld et al., 2018). Ces études corroboreraient le fait qu'il est généralement admis que les producteurs ont une aversion au risque. Shiferaw et al., (2009) ont quant à eux regrouper ces risques en risques liés aux chocs sanitaires, climatiques et socio-économiques. Les risques peuvent donc aussi être tant endogènes comme qu'exogènes. Les résultats de l'étude de Teklewold and Kohlin, (2011) ont montré que la majorité des producteurs se retrouvait dans la catégorie d'aversion au risque intermédiaires que sévère et extrême. Un degré élevé d'aversion au risque a un effet négatif car elle a abaissé l' adoption de la CS (Teklewold and Kohlin, 2011).

La perception des producteurs a joué un rôle très capital dans l'adoption de certaines technologies de GDT (Shiferaw, 1998; Vidogbéna et al., 2016). Il a été rapportée que la perception sur l'impact à long terme de l'AC (Chalak et al., 2017), la perception de la sécurité du régime foncier (Belay and Bewket, 2013), la perception des attributs propres à la technologie (Shiferaw, 1998), la perception de la menace de l'érosion (Shiferaw, 1998), le risque perçu d'investissement dans les terres en raison d'un conflit local (Rahman et al., 2017), la perception du risque de perte à la récolte (Thierfelder et al., 2015), ont une incidence sur l'adoption des technologies de GDT.

\section{Facteurs économiques}

Les facteurs économiques concernent les revenus générés par l'activité champêtre, les revenus hors-ferme soit l'emploi, l'épargne sur pied comme la possession d'actifs 
agricoles, de bétail, la possession de matériel et équipement agricole et les moyens de subsistance.

En tête des facteurs économiques nous avons le cheptel avec 14\% ( $\mathrm{n}=15)$ des articles considérés (Figure 4). Cela constitue une épargne sur pied en agriculture en milieu rural favorisant un investissement des producteurs dans l'adoption des technologies de GDT. Plusieurs études ont montré un rôle très contrasté du cheptel dans l'adoption des technologies de GDT (Caviglia-Harris, 2003; Pender and Gebremedhin, 2007; Belay and Bewket, 2013; Tesfaye et al., 2014; KabuboMariara, 2015; Asfaw et al., 2016; Etongo et al., 2018; Teshager Abeje et al., 2019). Le cheptel sert de banque pour les producteurs de ce milieu. C'est également un proxy pour évaluer le niveau de prospérité (Asfaw et al., 2016), qui est aussi le facteur clé de richesse incitant les producteurs à adopter les technologies d'intensification durable.

Le matériel agricole comme le tracteur ou la charrue sont compatibles avec l'adoption de certaines technologies de CES (Bodnár and De Graaff, 2003; Clay et al., 2014; Kabubo-Mariara, 2015; Ashoori et al., 2016; Lokonon and Mbaye, 2018; Issahaku and Abdul- Rahaman, 2019). La mécanisation a facilité l'adoption de technologies de conservation des ressources telles que le labour de conservation (Gollin et al., 2005). Les équipements agricoles pourraient toutefois être utilisés pour aider à la construction de structures de CS ou pour appliquer des intrants contribuant à prévenir l'érosion des sols, l'épuisement des nutriments ou d'autres formes de dégradation. Seulement $6 \%$ des articles $(\mathrm{n}=6)$ ont abordé ce facteur (Figure 4).

L'incitation à investir dans les terres est aussi influencée par les objectifs multiples des agriculteurs qui poursuivent plusieurs stratégies de subsistance (Jones, 1997; Pender, 2004; Cordingley et al., 2015; Lokonon and Mbaye, 2018; Teshager Abeje et al., 2019). Environ $6 \%$ des articles $(n=6)$ traitent de ce facteur. Les stratégies de développement rural durable et de gestion des terres dépendent des avantages comparatifs existant pour les populations. Les technologies de gestion durable des terres sont plus rapidement adoptées là où les activités de production à forte valeur ajoutée sont mise en œuvre (Pender, 2004). Par contre les résultats des travaux de Teshager Abeje et al., (2019) ont montré qu'avoir une grande diversification des moyens de subsistance a réduit l'opportunité d'adopter des technologies de GDT.

L'accès au revenu hors ferme (Pender and Gebremedhin, 2007; Wossen et al., 2013; Nigussie et al., 2017; Cholo et al., 2018; Martey et al., 2019) est aussi un avantage important dans l'adoption de technologie de GDT. L'emploi en milieu urbain (KabuboMariara, 2015) a permis aussi de capter des ressources financières pour investir dans la gestion durable des terres. Mais lorsque le coût d'opportunité dans les activés hors ferme s'avère plus élevé, cela devient un facteur négatif car les producteurs préfèrent aller travailler hors de leur ferme au détriment du maintien de la fertilité de leur sol. Le travail hors ferme aussi joue un rôle dans l'intensité de l'adoption de certaines technologies de GDT. Un cas empirique en Afrique de l'ouest a confirmé cela. Il a été démontré que la participation aux activités hors ferme était positivement corrélée à l'intensité de l'adoption des diguettes de pierre et de terre et de la fumure organique au Ghana (Issahaku and Abdul-Rahaman, 2019). Cela s'expliquerait par les revenus hors-ferme générés par ces activités.

L'accès aux input et équipements agricoles (herbicides, main d'œuvre, engrais, moyen de transport des inputs et output) (Bodnár and De Graaff, 2003; Teklewold et al., 2013; Asfaw et al., 2016; Ashoori et al., 2016 Nigussie et al., 2017; Etsay et al., 2019)a permis l'adoption de certaines technologies de GDT spécialement aux toutes premières années de l'adoption. Le nombre d'actif agricole (4\% des articles soit $\mathrm{n}=4$ ) (Pender and Gebremedhin, 2007; Cholo et al., 2018; Lokonon and Mbaye, 2018; Nigussie et al., 2017) représente un proxy du niveau de richesse dans certains villages et a joué également un rôle crucial dans l'adoption des technologies de GDT.. 
Le revenu a aussi joué un rôle capital dans l'adoption de technologies de GDT (Caviglia-Harris, 2003; Lee, 2005; Ashoori et al., 2016; Gao et al., 2018). Un haut revenu a encouragé l'adoption de technologies de conservation (Ashoori et al., 2016) parce qu'un revenu élevé permet de faire face aux dépenses liées aux opérations culturales et à la mise en place des technologies onéreuses. Très peu de publication ont traité de ce facteur ( $4 \%$ des articles soit $\mathrm{n}=4$ ). Le niveau de richesse aussi a contribué à l'adoption des technologies de GDT (Asfaw et al., 2016).

Plusieurs raisons économiques expliquent l'adoption et la dés-adoption des technologies de GDT. Les motivations les plus fréquemment mentionnées par les utilisateurs des terres pour adopter une technologie de GDT donnée sont globalement d'ordre économique: la production (24\%), une rentabilité accrue $(20 \%)$ et le bien-être et l'amélioration des moyens de subsistance (20\%). En outre, une charge de travail réduite a été mentionnée dans 5\% des cas (Giger et al., 2018). Les objectifs visés par les producteurs lors de l'adoption, ne sont atteignables qu'en réduisant les coûts d'investissement dans les GDT. Le challenge consiste à développer des technologies qui augmentent la valeur de la production agricole et / ou réduisent les coûts des intrants (Shepherd and Soule, 1998) mais cette étude a précisé que le défi concerne plus les exploitations dotées faiblement et moyennement en ressources. Le constat fait est que le rapport coût/avantage de la technologie devient négatif si les coûts d'investissements initiaux sont de plus en plus élevés. Les coûts d'établissement médians sont plus élevés en Europe comparés à l'Asie et l'Afrique. Cette différence bien que prévisible est probablement due principalement à des coûts de maind'œuvre plus élevés dans la zone européenne (Giger et al., 2018). Dans 43\% des cas, les coûts d'établissement étaient entièrement à la charge des agriculteurs eux-mêmes (Giger et al., 2018), ce qui freine l'adoption de plusieurs technologies de GDT. Les producteurs ne sont pas motivés à adopter quand l'investissement initial ne couvre pas les avantages à moyen terme.

\section{Facteurs institutionnels}

L'ancrage institutionnel est aussi important dans la décision d'adoption d'une technologie de GDT. Les facteurs institutionnels concernent le contact avec les institutions de vulgarisation, la sécurité foncière, l'accès au crédit, l'assurance, les institutions de GRN et le soutien du gouvernement. Ces facteurs ont la particularité de mettre les producteurs en contact avec des institutions au niveau méso.

Le facteur institutionnel le plus largement cité est la sécurité foncière. Elle est abordée par 21\% ( $\mathrm{n}=30)$ des articles (Figure 5). La sécurité foncière est un facteur capital favorisant l'adoption des technologies de GDT (Viswanath et al., 2000; Waswa et al., 2002; Lee, 2005; Ajayi et al., 2007; Saïdou et al., 2007;Bond, 2009; Shiferaw et al., 2009; Banadda, 2010; Teklewold and Kohlin, 2011; Wossen et al., 2013; Kabubo-Mariara, 2015; Adimassu et al., 2016; Asfaw et al., 2016; Nigussie et al., 2017; Rahman et al., 2017; Tey et al., 2017, Lokonon and Mbaye, 2018;Nkomoki et al., 2018; Etongo et al., 2018; Issahaku and Abdul-Rahaman, 2019). La sécurité foncière est définie comme l'interaction entre le contenu de la tenure (droit fort sur le droit de vente de la terre) et les aspects de l'assurance liés à la terre (perception d'une faible probabilité d'expropriation) (Kabubo-Mariara, 2015). Lorsque les occupants disposent de l'usufruit sur les terres occupées, ces derniers décident d'adopter des technologies dont le retour sur investissement est étalonné sur le moyen ou le long terme à l'instar de l'agroforesterie mais par contre adoptent des mesures agronomiques s'ils ne disposent pas d'un droit sur la terre. Ce dernier cas de figure peut contribuer à dégrader les terres agricoles parce que conduisant à une agriculture minière. Cependant la sécurité foncière n'est pas toujours gage d'investissement dans l'amélioration des terres (Waswa et al., 2002). En d'autres termes les producteurs pauvres qui n'arrivent pas à avoir les droits de propriétés pour sécuriser les terres, peuvent voir leur horizon de planification réduite dans le but d'améliorer les terres agricoles. Très souvent ces producteurs ont les 
droits d'utilisation et non l'usufruit. De plus, la sécurité foncière par l'acquisition des droits de propriété peut favoriser l'accès au crédit agricole permettant aux producteurs d'investir dans les technologies de GDT. Ceci expliquerait le fait que l'insécurité foncière a démotivé l'adoption de technologie d'intensification durable (Rahman et al., 2017).

Le contact avec un service de vulgarisation comme révélé par certaines études (Paudel and Thapa, 2004; Pender and Gebremedhin, 2007; Teklewold and Kohlin, 2011; Wossen et al., 2013; Mulale et al., 2014;Ndiritu et al., 2014; Kabubo-Mariara, 2015; Asfaw et al., 2016; Nigussie et al., 2017;Rahman et al., 2017; Hassen, 2018; Zeweld et al., 2018; Etsay et al., 2019; Issahaku and Abdul-Rahaman, 2019) a joué un rôle important dans l'adoption de technologies de GDT. Par ce contact, les agriculteurs peuvent obtenir des informations sur de meilleures technologies agricoles et améliorent leur compréhension et capacité technique de la CS par exemple. Le rôle de ces institutions de vulgarisation peuvent s'avérer parfois aussi négatif en dissuadant les agriculteurs d'adopter les technologies par la mauvaise qualité de service fourni ou parfois leur action coercitive (Mulale et al., 2014, Tougiani et al, 2009).

L'accès au crédit agricole a été rapporté dans plusieurs études menées dans plusieurs régions du monde comme un facteur clé favorisant l'adoption de technologies de GDT (Caviglia-Harris, 2003; Pender and Gebremedhin, 2007; Ndiritu et al., 2014; Tesfaye et al., 2014; Asfaw et al., 2016; Nigussie et al., 2017; Nkomoki et al., 2018; Zeweld et al., 2018; Teshager Abeje et al., 2019; Etsay et al., 2019). Le crédit permet de lever la contrainte capitale pour l'investissement dans les technologies dont les coûts initiaux de réalisation sont souvent élevés. Les producteurs y accèdent plus facilement s'ils procurent la preuve de leur adhésion formelle à une organisation de producteur ou parfois s'ils peuvent fournir une attestation de détention de droits de propriété de leur terre (sécurité foncière).

La soumission à une assurance permet de lever plusieurs barrières financières à l'adoption de technologies de GDT. Une étude en Chine a corroboré cela (Gao et al., 2018); une assurance agricole est donc un moteur positif d'adoption.

L'appartenance à une organisation formelle ou informelle influence l'adoption des technologies de GDT (Wossen et al., 2013; Asfaw et al., 2016; Gao et al., 2018; Lokonon and Mbaye, 2018; Nkomoki et al., 2018; Zeweld et al., 2018; Issahaku and AbdulRahaman, 2019; Razzaghi Borkhani and Mohammadi, 2019). L'effet de l'appartenance à une organisation informelle a joué un rôle négatif dans l'adoption de technologie de GDT (Wossen et al., 2013) à cause des problèmes de free-riding à l'intérieur des groupes ou d'autres se cachent sur les efforts des autres pour capter des avantages liés aux ressources naturelles en open accès, tout comme ce signe négatif pourrait venir de l'influence de certaines personnes qui usent de leur influence dans le groupe au détriment des autres. Le signe positif de l'appartenance à une organisation formelle (Wossen et al., 2013) proviendrait du fait que cette appartenance sociale est le gage de l'accès au crédit agricole et par ricochet à certains intrants agricoles source d'une intensification durable de l'agriculture. D'autres études ont mis en relief le nombre d'organisation auquel participe l'agriculteur (Caviglia-Harris, 2003) comme étant le moteur de l'adoption de technologie durable. Lorsque l'organisation est bien formalisée et dispose d'une certaine autonomie, elle peut fournir des services d'éducation par rapport au marché ou même sur l'opérationnalisation des itinéraires techniques de production (Caviglia-Harris, 2003).

Le soutien du gouvernement peut s'avérer être un facteur important dans l'adoption des technologies de GDT (Shiferaw et al., 2009; Teklewold et al., 2013; Ndiritu et al., 2014; Cortner et al., 2019). Les agriculteurs qui sont pour la plupart pauvres en milieu rural profitent de ce soutien du gouvernement pour alléger les coûts d'investissement initiaux qui ne sont pas toujours évidents à supporter par ces derniers. Cependant très peu de publications l'ont mentionné avec une 
proportion de $4 \% \quad(n=4)$ (Figure 5). Les producteurs ayant participé à un essai de démonstration étaient plus susceptibles d'adopter la technologie étudiée (Ahaneku, 2010; Vidogbéna et al., 2016; Chalak et al., 2017; Nigussie et al., 2017b).

\section{Facteurs biophysiques}

Les facteurs biophysiques concernent les caractéristiques de la parcelle, la taille de la parcelle, la localisation de la parcelle, les facteurs climatiques (la pluie), les essais de démonstration.

Le facteur biophysique le plus cité est la taille de la parcelle ou de la superficie disponible avec $13 \%$ d'apparition dans les publications. Certaines technologies dont la réalisation demande un vaste espace expliqueraient cela (Montagnini and Finney, 2011; Teklewold and Kohlin, 2011; Teklewold et al., 2013; Branca et al., 2013; Schmidt et al., 2017). La gestion de l'eau est cruciale dans l'adoption de certaines technologies de GDT.

L'eau est un paramètre important dans l'adoption de certaines technologies de GDT. La pluie a été abordée par 9\% $(\mathrm{n}=10)$ des articles (Figure 6). La pluviométrie a été mentionnée comme capable d'inciter l'adoption d'une technologie ou non (Shiferaw et al., 2009; Branca et al., 2013; Teklewold et al., 2013; Kabubo-Mariara, 2015; Asfaw et al., 2016; Gao et al., 2018; Hassen, 2018; Cholo et al., 2018; Cortner et al., 2019; Issahaku and Abdul-Rahaman, 2019). Une pluviométrie élevée rend l'eau disponible et incite à l'utilisation de technologies agronomiques comme l'utilisation des engrais minéraux. Cela favorise les zones a fort stress hydrique de voir leur semis ou cultures ne pas mourir (bruler) en terres acides. L'eau est cependant négativement corrélée avec l'abandon de l'utilisation de technologie agricole issue de la révolution verte (Hassen, 2018). La proximité d'un lac ou ruisseau/rivière a conditionné l'adoption de certaines technologies de GDT (Lokonon and Mbaye, 2018); c'est d'ailleurs le seul document qui relate cela. L'accès aux eaux d'irrigation a incité positivement l'adoption des technologies de GDT (Pender and Gebremedhin, 2007; Chalak et al., 2017; Lokonon and Mbaye, 2018; Etsay et al., 2019). Certains producteurs utilisent ces eaux d'irrigation pour arroser les arbres fruitiers en technologie d'agroforesterie en saison sèche. La température (Asfaw et al., 2016; Cholo et al., 2018; Gao et al., 2018) aussi a eu un effet similaire.

Les caractéristiques de la parcelle peuvent motiver ou décourager les agriculteurs à adopter les technologies de GDT (Ajayi et al., 2007; Asfaw et al., 2016; Nigussie et al., 2017; Tey et al., 2017; Abi et al., 2018; Hassen, 2018). Le type de terrain a influencé grandement la décision d'adoption des agriculteurs (Asfaw et al., 2016). Une pente plus plate de la parcelle (Hassen, 2018) a incité positivement les agriculteurs a adopté des technologies de GDT contrairement aux parcelles escarpées (Abi et al., 2018), souvent sujets à l'érosion hydrique ou aux parcelles plus abruptes (Tey et al., 2017). Les terres fertiles sont aussi un facteur déterminant l'investissement dans les terres (Abi et al., 2018; Kabubo-Mariara, 2015). Les agriculteurs adoptent des technologies de GDT sur les zones moins érosives et fertiles.

La taille de la parcelle, de la ferme ou de l'exploitation selon la nomenclature utilisée a été mentionnée aussi comme influençant la décision des producteurs (Pender and Gebremedhin, 2007; Clay et al., 2014; Tesfaye et al., 2014; Obayelu et al., 2016; Asfaw et al., 2016; Ashoori et al., 2016; Tey et al., 2017; Nigussie et al., 2017; Gao et al., 2018; Lokonon and Mbaye, 2018; Nkomoki et al., 2018; Etongo et al., 2018; Etsay et al., 2019; Issahaku and Abdul-Rahaman, 2019; Martey et al., 2019). La taille d'une ferme est importante pour la prise de décision en matière de GDT (Etongo et al., 2018). Les résultats rapportés par l'étude de Etongo et al. (2018) ont montré par exemple que la construction de fosses à planter nécessitant une forte intensité de maind'œuvre dépendait de la taille de la parcelle. Par 
contre, c'est la taille de superficie cultivable disponible qui a été évoquée au Liban (Ashoori et al., 2016). Les technologies de GDT physiques prennent plus d'espace. Une grande superficie disponible motive l'adoption de technologies qui sont demandeuses de grande espace dans leurs installations.

L'accès à la route peut contribuer à adopter certaines technologies de GDT (Pender and Gebremedhin, 2007; Asfaw et al., 2016; Ashoori et al., 2016). Ce résultat suggère que l'amélioration de l'accès aux routes peut favoriser une gestion intensive des terres, alors que la fragmentation des terres (plusieurs parcelles éloignées) peut la compromettre. Des simulations faites sur l'accès aux routes en supposant que $10 \%$ des routes en terre soient modernisées et gravelées, ont entrainé une réduction de $30 \%$ des coûts de transport liés aux produits ce qui est important sur le revenu de l'agriculteur (Schmidt et al., 2017) et ont favorisé l'adoption de technologies de GDT.

La distance de la maison de l'agriculteur à la route principale (Ashoori et al., 2016; Lokonon and Mbaye, 2018) a favorisé ou démotivé l'adoption de technologies de GDT. Cela s'expliquerait par le fait que ce facteur représente un proxy pour l'accès aux intrants agricoles nécessaires à la mise en œuvre des technologies de GDT. Une autre explication est que les producteurs qui vivent près de la route sont plus favorisés pour recevoir la visite des agents de vulgarisation et donc de recevoir des informations utiles à l'adoption des technologies de GDT. La distance de la maison du producteur au marché (Caviglia-Harris, 2003; Asfaw et al., 2016; Lokonon and Mbaye, 2018) est un proxy de l'accès au marché (Pender and Gebremedhin, 2007). Les politiques de prix et les conditions du marché ont façonné les modèles d'adoption de certaines technologies (Gollin et al., 2005).

La proximité de la parcelle aux zones de résidence des producteurs (Pender and Gebremedhin, 2007; Belay and Bewket, 2013; Teklewold et al., 2013; Kabubo-Mariara, 2015 Abi et al., 2018; Etsay et al., 2019) a été significative dans le choix d'adoption de certaines technologies de GDT. Les parcelles situées non loin des maisons bénéficient d'attention particulière concernant la gestion de la fertilité des sols. Les producteurs adoptent des technologies de GDT sur ces terres. Par contre quand la parcelle est éloignée, les producteurs ne sont pas enclins à investir dans la fertilité de ces terres. C'est aussi un indicateur de fragmentation de la terre. Plus les terres sont fragmentées moins les producteurs adoptent des technologies de GDT (Cholo et al, 2018).

Le nombre d'année d'exploitation d'une parcelle (Caviglia-Harris, 2003; Etongo et al., 2018) peut aussi déterminer ou non l'adoption de technologies propices à ces types de parcelles. Aussi longtemps la terre est occupée, cela devient un gage pour les générations futures et donc les ayant droits du producteur pourraient continuer à utiliser cette parcelle. Cela favorise ainsi l'adoption de technologie qui s'étende sur un moyen ou long terme.

L'aire géographique ou la région d'étude s'est révélée comme un facteur d'adoption de technologies de GDT(Shiferaw, 1998; Bodnár and De Graaff, 2003; Ajayi et al., 2007; Kabubo-Mariara, 2015; Asfaw et al., 2016; Etsay et al., 2019; Teshager Abeje et al., 2019). Le lieu de résidence des producteurs peut déterminer la nature de certains résultats par rapport à d'autre. Au Mali, les agriculteurs de la zone cotonnière ont par exemple adopté moins de mesures de CES (Bodnár and De Graaff, 2003).

\section{Genre et GDT}

Le genre a été très peu étudié en GDT dans le domaine des sciences sociales. Seulement $4 \%$ des publications ont abordé le genre en relation avec les technologies de GDT (Bertin et al., 2014; Ndiritu et al., 2014; Kondylis et al., 2016; Emerton and Snyder, 2018). Cette étude révèle l'existence des inégalités socioéconomiques qui ont une incidence sur les ménages qui ont particulièrement une femme à leur tête. Les résultats de Emerton et Snyder, (2018) ont 
abondé dans ce sens en ce qu'ils ont conclu que le revenu des hommes était supérieur à celui des femmes. Des résultats empiriques ont montré qu'il n'y a pas de différence selon le genre pour l'utilisation de l'engrais minéral, la CES, la rotation maïs-légumineuse, l'association culturale maïs-légumineuse et l'utilisation des semences améliorées mais les femmes adoptaient moins le fumier et le minimum labour (Ndiritu et al., 2014). Pour pallier à l'inégalité du genre, Bertin et al., (2014) en Afrique centrale ont milité pour la participation des femmes aux approches de vulgarisation agricole. Cela a conduit à une amélioration du nombre de femmes dans l'adoption des technologies de GDT. Ils appellent à des technologies qui sont plus appropriées pour les femmes de façon à ce qu'elles soient adoptées autant par les femmes que les hommes. Une autre façon d'aborder le genre est dans la méthode de collecte des données. Pour des raisons d'ordre culturelles, il serait peut-être préférable d'utiliser des femmes pour conduire les entretiens avec les femmes dans le but d'avoir des informations de qualité (Dessie et al., 2012) qui pourraient avoir une incidence dans les différents indicateurs de genre souvent utilisés. En effet, devant une personne de sexe opposé, certaines femmes sont réticentes à donner certaines informations très sensibles.

\section{Type d'articles}

Article de recherche $\quad$ Article de synthèse

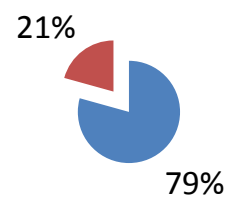

Figure 1 : Type d'articles.

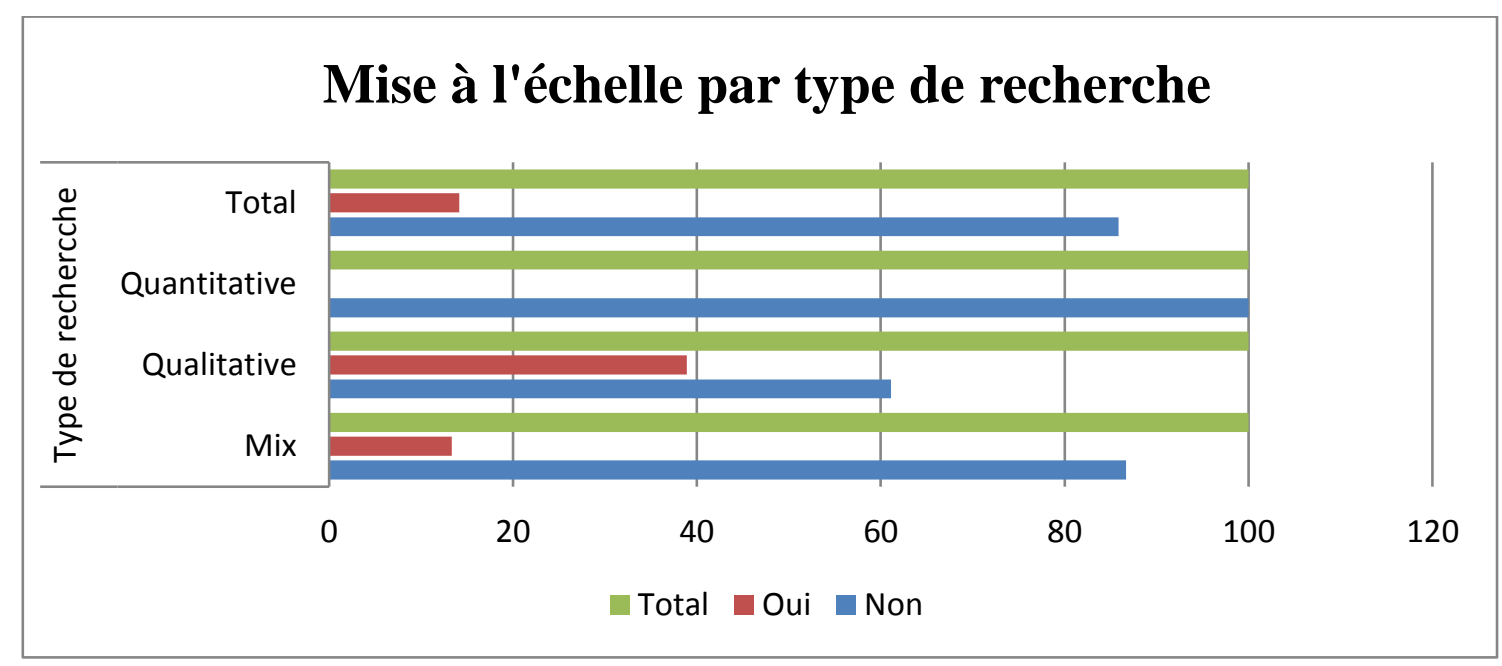

Figure 2: Mise à l'échelle par type de recherche. 


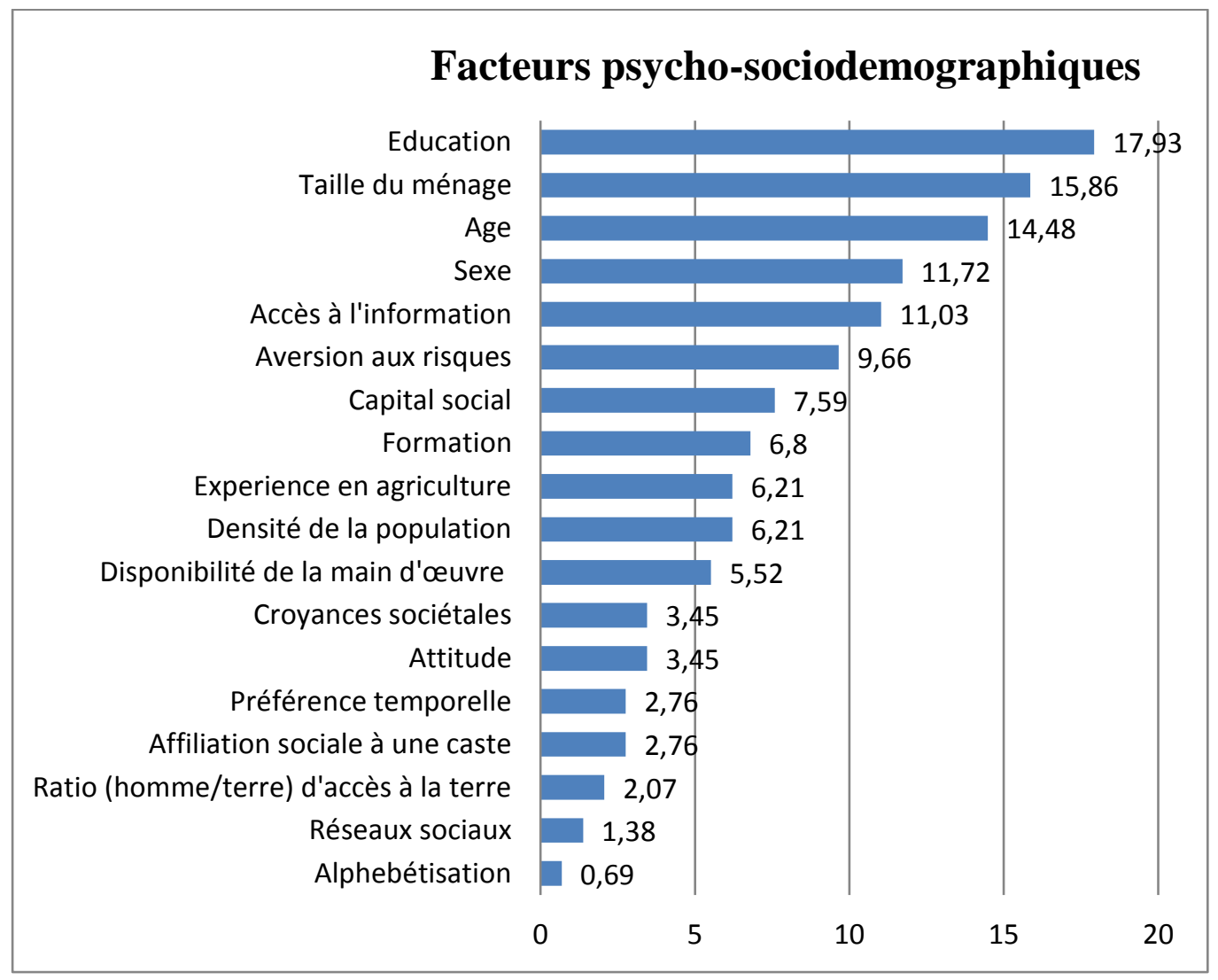

Figure 3 : Facteurs psycho-sociodémographiques.

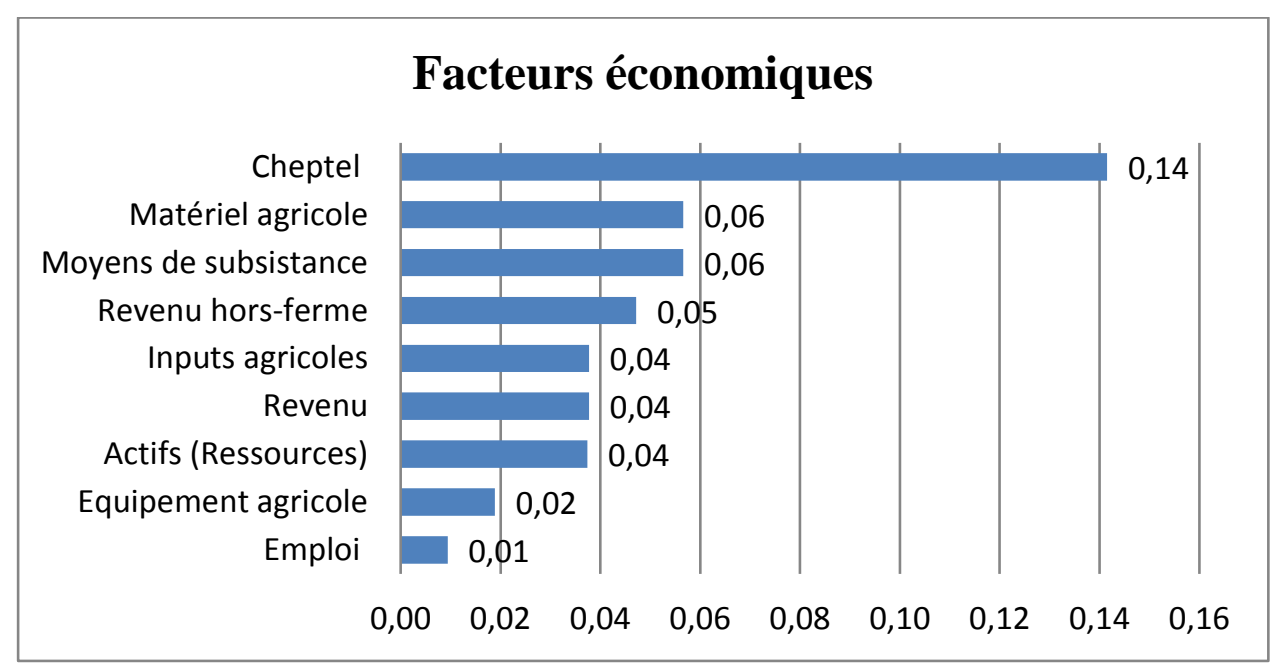

Figure 4 : Facteurs économiques. 


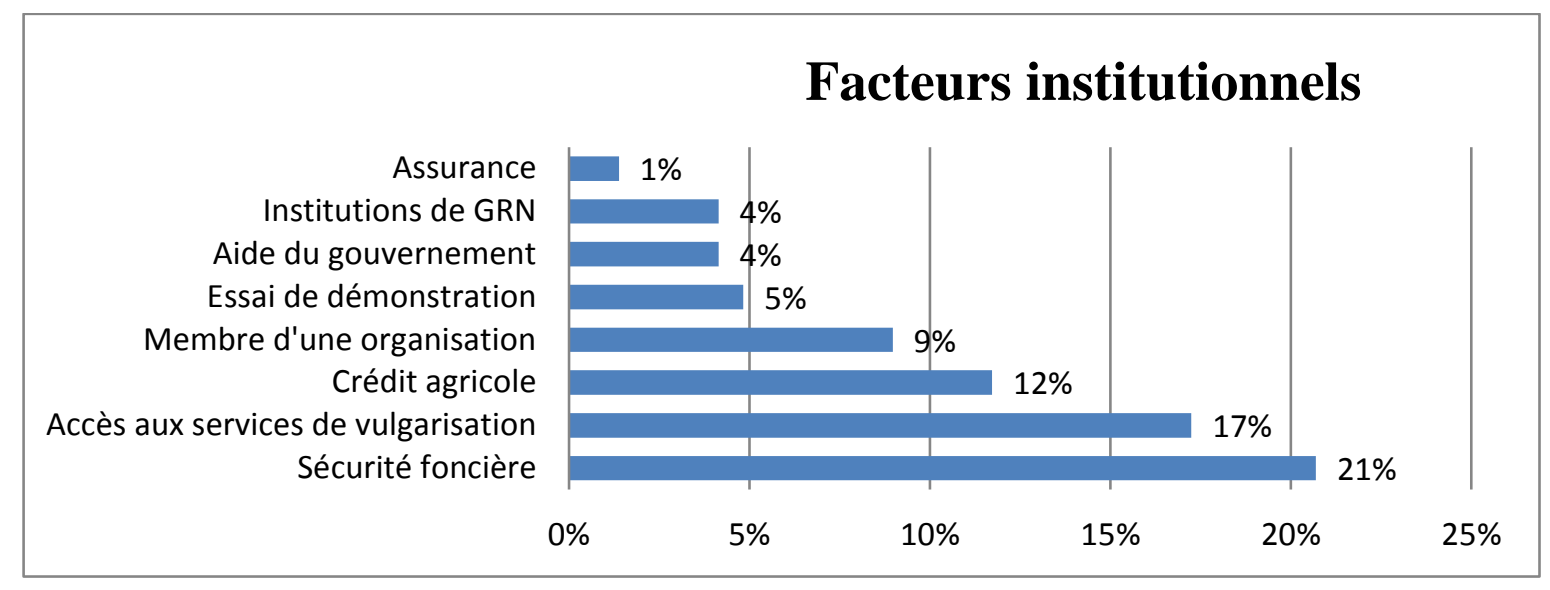

Figure 5 : Facteurs institutionnels.

\section{Facteurs biophysiques}

Taille de la parcelle

Pluie

Caractéristiques de la parcelle

Distance de la maison du producteur à la parcelle

Distance de la maison du producteur au marché

Irrigation

Accessibilité à la voie

Parcelle non éloigné de la maison

Nombre d'années d'utilisation de la parcelle

Rivière à coté

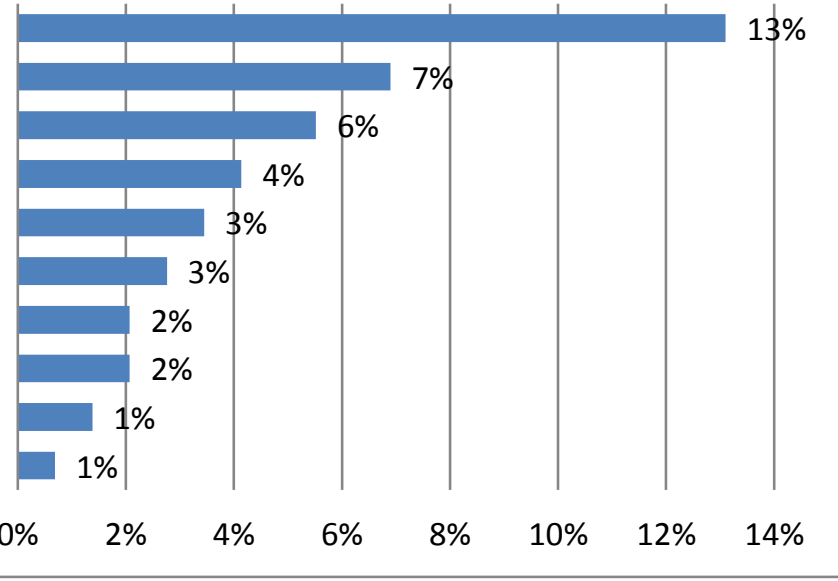

Figure 6 : Facteurs biophysiques.

\section{CONCLUSION}

Cet article a passé en revue et synthétisé les études récentes sur les technologies de GDT dans le but d'identifier les facteurs qui influencent la décision d'adoption et les approches de mise à l'échelle dans les PEVD, dans le but de savoir les leviers sur lequel il faut agir pour améliorer les taux d'adoption. Cette revue a identifié plusieurs facteurs déterminants l'adoption des technologies GDT.

Ces facteurs sont regroupés en 4 sous-groupes. Premièrement, les facteurs psycho- sociodémographiques ; deuxièmement les facteurs institutionnels qui ne sont pas liés aux producteurs directement et situés à un niveau méso ; troisièmement les facteurs économiques qui ont un caractère monétaire et enfin en quatrième lieu, les facteurs biophysiques qui ont rapport avec l'environnement de la parcelle. Au vue de tous ces facteurs, il convient de mettre en lumière ceux qui attirent facilement l'attention parce qu'ils sont en tête de ces sous-groupes à savoir i) l'éducation ii) le cheptel iii) la sécurité foncière et iv) la taille de la parcelle. Cependant le rôle de certains de 
ces facteurs reste ambigu et une méta-analyse sur chacun de ces facteurs pourrait être capitale pour mieux comprendre comment formuler des politiques agricoles à l'endroit des petites exploitations de ces PEVD dans le but d'améliorer leur bien-être. Aussi, l'éducation et la sécurité foncière dégage un consensus clair en ce sens qu'ils favorisent l'adoption des technologies de GDT. Il faudra développer des programmes d'alphabétisation pour accompagner les programmes de GDT et aussi enclencher un processus de sécurisation des terres pour favoriser des investissements à long terme sur ces parcelles.

Avant l'adoption, il urge de trouver un bon canal de diffusion de ces technologies de GDT. C'est pourquoi cette revue et synthèse a aussi mis l'accent sur la nécessité d'impliquer les producteurs depuis le design de la technologie jusqu'à sa mise à l'échelle. Les programmes de GDT qui négligent cet aspect ont connu de fort taux d'échec et il urge de rendre vraiment effectif la participation des producteurs dans le processus de diffusion tout en tenant compte de l'aspect genre dans leur mise en œuvre.

\section{CONFLIT D'INTERETS}

Aucun conflit d'intérêts n'est à signalé concernant ce document.

\section{CONTRIBUTIONS DES AUTEURS}

STCA a conçu la base de données, analysé les données, rédigé l'article, YPA a défini la méthodologie qui a permis de collecter les données et JAY a corrigé l'article.

\section{REMERCIEMENTS}

Nous remercions Dr Rosaine YEGBEMEY pour ces conseils dans l'orientation à donner aux analyses faite lors de l'élaboration de ce document. Egalement nous remercions AFFOUKOU Kévin, MOUTOUAMA Jacob, AIHOUTON Ghislain, KIRABE ALLOU Bio Ganni et HOUNTONDJI S. Paul pour leur contribution à la conception de la base de données de documents ayant permis la réalisation de cet article.

\section{REFERENCES}

Abi M, Kessler A, Oosterveer P, Degaga D. 2018. Understanding the Spontaneous Spreading of Stone Bunds in Ethiopia: Implications for Sustainable Land Management. https://doi.org/10.3390/su10082666

Adebiyi KD, Maiga-yaleu S, Issaka K, AYENA M, Yabi JA. 2019. Déterminants de l'adoption des bonnes pratiques de gestion durable des terres dans un contexte de changement climatique au Nord Bénin : cas de la fumure organique. Int. J. Biol. Chem. Sci., 13(2): 998-1010. DOI: https://dx.doi.org/10.4314/ijbcs.v13i2.34

Adimassu Z, Langan S, Johnston R. 2016. Understanding determinants of farmers' investments in sustainable land management practices in Ethiopia: Review and synthesis. Environment, Development and Sustainability, $\mathbf{1 8}$ 1005-1023. DOI: https://doi.org/10.1007/s10668-0159683-5

Ahaneku IE. 2010. Conservation of soil and water resources for combating food crisis in Nigeria. Scientific Research and Essays, 5: 507-513.

Ajayi OC, Akinnifesi FK, Sileshi G, Chakeredza S. 2007. Adoption of renewable soil fertility replenishment technologies in the southern African region: Lessons learnt and the way forward: Oluyede Clifford Ajayiet al. /Natural Resources Forum 31 (2007) 306317. Natural Resources Forum, 31: 306317. DOI: https://doi.org/10.1111/j.14778947.2007.00163.

Amede T, Kassa H, Zeleke G, Shiferaw A, Kismu S, Teshome M. 2007. Working with communities and building local institutions for sustainable land management in the Ethiopian highlands. Mountain Research and Development, 27: 15-20. DOI: https://doi.org/10.1659/02764741(2007)27[15:WWCABL]2.0.CO;2

Asfaw S, McCarthy N, Lipper L, Arslan A, Cattaneo A. 2016. What determines farmers' adaptive capacity? Empirical evidence from Malawi. Food Security, 8 : 
643-664.

https://doi.org/10.1007/s12571-0160571-0

Ashoori D, Allahyari MS, Damalas CA. 2016. Adoption of conservation farming practices for sustainable rice production among small-scale paddy farmers in northern Iran. Paddy and Water Environment, 15: 237-248. DOI: https://doi.org/10.1007/s10333-0160543-1

Banadda N. 2010. Gaps, barriers and bottlenecks to sustainable land management (SLM) adoption in Uganda. African Journal of Agricultural Research, 5: 3571-3580. DOI: 10.5897/AJAR10.029

Bazame R, Tanrıvermis H, Kapusuz YE. 2019. Land management and sustainable use of land resources in the case of Burkina Faso. Land Degradation \& Development, 30: 608-621. DOI: https://doi.org/10.1002/ldr.3247

Belay M, Bewket W. 2013. Farmers' livelihood assets and adoption of sustainable land management practices in north-western highlands of Ethiopia. International Journal of Environmental Studies, 70: 284-301. DOI: https://doi.org/10.1080/00207233.2013.7 74773

Bertin T, Zacharie T, Ann D, Ebenezar A, Alain T. 2014. Scaling-up sustainable land management practices through the concept of the rural resource centre: reconciling farmers' interests with research agendas. The Journal of Agricultural Education and Extension, 20: $463-483 . \quad$ DOI: 10.1080/1389224X.2014.913984

Blake WH, Rabinovich A, Wynants M, Kelly C, Nasseri M, Ngondya I, Patrick A, Mtei K, Munishi L, Boeckx P, Navas A, Smith HG, Gilvear D, Wilson G, Roberts N, Ndakidemi P. 2018. Soil erosion in East Africa: an interdisciplinary approach to realising pastoral land management change. Environmental Research Letters, 13: $124014 . \quad$ DOI: https://doi.org/10.1088/1748-

9326/aaea 8 b
Bodnár F, De Graaff J. 2003. Factors influencing adoption of soil and water conservation measures in southern Mali: INFLUENCES ON FARMERS' ADOPTION OF SWC MEASURES. Land Degradation \& Development, 14: 515-525. DOI: https://doi.org/10.1002/ldr.579

Bond AC. 2009. Contextual Analysis of Agroforestry Adoption in the Buffer Zone of Podocarpus National Park, Ecuador. Journal of Sustainable Forestry, 28: 825843.

DOI: https://doi.org/10.1080/10549810902794 568

Branca G, Lipper L, McCarthy N, Jolejole MC. 2013. Food security, climate change, and sustainable land management. A review. Agronomy for Sustainable Development, 33: 635-650. DOI : 10.1007/s13593-0130133-1

Carriazo F, Labarta R, Escobedo FJ. 2020. Incentivizing sustainable rangeland practices and policies in Colombia's Orinoco region. Land Use Policy, 95: 104203.

DOI: https://doi.org/10.1016/j.landusepol.2019 .104203

Caviglia-Harris JL. 2003. Sustainable agricultural practices in Rondonia, Brazil: Do local farmer organizations affect adoption rates? Economic Development and Cultural Change, 52: 23-49.

Chalak A, Irani A, Chaaban J, Bashour I, Seyfert K, Smoot K, Abebe GK. 2017. Farmers Willingness to Adopt Conservation Agriculture: New Evidence from Lebanon. Environmental Management, 60: 693-704. DOI: https://doi.org/10.1007/s00267-0170904-6

Chinseu E, Dougill A, Stringer L. 2019. Why do smallholder farmers dis-adopt conservation agriculture? Insights from Malawi. Land Degradation \& Development, 30: 533-543. DOI: https://doi.org/10.1002/ldr.3190

Cholo TC, Fleskens L, Sietz D, Peerlings J. 2018. Is Land Fragmentation Facilitating or Obstructing Adoption of Climate Adaptation Measures in Ethiopia? 
Sustainability, 10: 2120. DOI: https://doi.org/10.3390/su10072120

Clay DE, Clay SA, Reitsma KD, Dunn BH, Smart AJ, Carlson GG, Horvath D, Stone JJ. 2014. Does the conversion of grasslands to row crop production in semi-arid areas threaten global food supplies? Global Food Security, 3: 22 30.

DOI: https://doi.org/10.1016/j.gfs.2013.12.002

Cools N, De Pauw E, Deckers J. 2003. Towards an integration of conventional land evaluation methods and farmers' soil suitability assessment: a case study in northwestern Syria. Agriculture, Ecosystems \& Environment, 95: 327342. DOI: $\quad 10.1016 / \mathrm{S} 0167$ 8809(02)00045-2

Cordingley JE, Snyder KA, Rosendahl J, Kizito F, Bossio D. 2015. Thinking outside the plot: Addressing low adoption of sustainable land management in subSaharan Africa. Current Opinion in Environmental Sustainability, 15: 35-40.

Cortner O, Garrett RD, Valentim JF, Ferreira J, Niles MT, Reis J, Gil J. 2019. Perceptions of integrated crop-livestock systems for sustainable intensification in the Brazilian Amazon. Land Use Policy, 82: 841-853. DOI: 10.1016/j.cosust.2015.07.010.

Dessalegn B, Kiktenko L, Zhumagazina B, Zhakenova S, Nangia V. 2018. Explaining farmers' reluctance to adopt recommendations for sustainable ecosystem management. Ecological Processes, $\quad 7$ : $1 . \quad$ DOI: https://doi.org/10.1186/s13717-0180133-9

Dessie Y, Wurzinger M, Hauser M. 2012. The role of social learning for soil conservation: the case of Amba Zuria land management, Ethiopia. International Journal of Sustainable Development \& World Ecology, 19: 258-267. DOI: https://doi.org/10.1080/13504509.2011.6 36082

Diao X, Sarpong DB. 2011. Poverty Implications of Agricultural Land Degradation in Ghana: An Economywide, Multimarket Model Assessment: Poverty Implications of Agricultural Land Degradation in Ghana. African
Development Review, 23: 263-275. https://doi.org/10.1111/j.1467-

8268.2011.00285.x

Drechsel P, Steiner KG, Hagedorn F. 1996. A review on the potential of improved fallows and green manure in Rwanda. Agroforestry Systems, 33: 109-136. DOI: 10.1007/BF00213645

Emerton L, Snyder KA. 2018. Rethinking sustainable land management planning: Understanding the social and economic drivers of farmer decision-making in Africa. Land Use Policy, 79: 684-694. DOI: 10.1016/j.landusepol.2018.08.041

Etongo D, Epule TE, Djenontin INS, Kanninen M. 2018. Land management in rural Burkina Faso: the role of socio-cultural and institutional factors. Natural Resources Forum, 42: 201-213. DOI: https://doi.org/10.1111/1477-8947.12153

Etsay H, Negash T, Aregay M. 2019. Factors that influence the implementation of sustainable land management practices by rural households in Tigrai region, Ethiopia. Ecological Processes, 8: 14. DOI: $10.1186 / \mathrm{s} 13717-019-0166-8$

Fleskens L, Nainggolan D, Stringer LC. 2014. An exploration of scenarios to support sustainable land management using integrated environmental socio-economic models. Environmental Management, 54: 1005-1021. DOI: 10.1007/s00267-0130202-x

Gao L, Zhang W, Mei Y, Sam AG, Song Y, Jin S. 2018. Do farmers adopt fewer conservation practices on rented land? Evidence from straw retention in China. Land Use Policy, 79: 609-621. DOI: 10.1016/j.landusepol.2018.08.026

Giger M, Liniger H, Sauter C, Schwilch G. 2018. Economic benefits and costs of sustainable land management technologies: an analysis of WOCAT's global data. Land Degradation \& Development, 29: 962-974. DOI: https://doi.org/10.1002/ldr.2429

Gollin D, Morris M, Byerle D. 2005. Technology Adoption in Intensive PostGreen Revolution Systems. American Journal of Agricultural Economics, 87: 1310-1316.

DOI: 
https://doi.org/10.1111/j.1467-

8276.2005.00824.x

Hassen S. 2018. The effect of farmyard manure on the continued and discontinued use of inorganic fertilizer in Ethiopia: An ordered probit analysis. Land Use Policy, 72: 523-532. DOI: 10.1016/j.landusepol.2018.01.002

Homann S, Rischkowsky B, Steinbach J, Kirk M, Mathias E. 2008. Towards endogenous livestock development: Borana pastoralists' responses to environmental and institutional changes. Human Ecology, 36: 503. DOI: 10.1007/s10745-008-9180-7

Issahaku G, Abdul-Rahaman A. 2019. Sustainable land management practices, off-farm work participation and vulnerability among farmers in Ghana: Is there a nexus? International Soil and Water Conservation Research, 7: 18-26. DOI:

https://doi.org/10.1016/j.iswcr.2018.10.0 02

Jones S. 1997. An actor-level analysis of the constraints on sustainable land management in northern Thailand: A study from Chiang Dao District. South East Asia Research, 5: 243-267. DOI: https://doi.org/10.1177/0967828X97005 00303

Kabubo-Mariara J. 2015. Does Institutional Isolation Matter for Soil Conservation Decisions? Evidence From Kenya. SAGE Open, 5: 215824401557097. DOI: https://doi.org/10.1177/21582440155709 77

Kohio EN, Touré AG, SEDOGO MP, Ambouta KJ-M. 2017. Contraintes à leadoption des bonnes pratiques de Gestion Durable des Terres dans les zones soudaniennes et soudano-sahéliennes du Burkina Faso. Int. J. Biol. Chem. Sci., 11(6): 2982-2989. DOI: https://dx.doi.org/10.4314/ijbcs.v11i6.34

Kondylis F, Mueller V, Sheriff G, Zhu S. 2016. Do Female Instructors Reduce Gender Bias in Diffusion of Sustainable Land Management Techniques? Experimental Evidence From Mozambique. World Development, $\quad$ 78: 436-449. DOI: https://doi.org/10.1016/j.worlddev.2015. 10.036

Kwayu EJ, Sallu SM, Paavola J. 2014. Farmer participation in the equitable payments for watershed services in Morogoro, Tanzania. Ecosystem Services, 7: 1-9. DOI: 10.1016/j.ecoser.2013.12.006

Lee DR. 2005. Agricultural Sustainability and Technology Adoption: Issues and Policies for Developing Countries. American Journal of Agricultural Economics, 87: 1325-1334. DOI: https://doi.org/10.1111/j.14678276.2005.00826.x

Lokonon BO, Mbaye AA. 2018. Climate change and adoption of sustainable land management practices in the Niger basin of Benin, in: Natural Resources Forum, Wiley Online Library: 42-53. DOI: https://doi.org/10.1111/1477-8947.12142

Magni G. 2017. Indigenous knowledge and implications for the sustainable development agenda. European Journal of Education, 52: 437-447. DOI: https://doi.org/10.1111/ejed.12238

Martey E, Kuwornu JK, Adjebeng-Danquah J. 2019. Estimating the effect of mineral fertilizer use on Land productivity and income: Evidence from Ghana. Land Use Policy, 85: 463-475. DOI: https://doi.org/10.1016/j.landusepol.2019 .04 .027

Montagnini F, Finney C. 2011. Payments for environmental services in Latin America as a tool for restoration and rural development. Ambio, 40: 285-297. DOI: 10.1007/s13280-010-0114-4

Mulale K, Chanda R, Perkins JS, Magole L, Sebego RJ, Atlhopheng JR, Mphinyane W, Reed MS. 2014. Formal institutions and their role in promoting sustainable land management in Boteti, Botswana. Land Degradation \& Development, 25: 80-91. DOI: $10.1002 / \mathrm{ldr} .2274$

Ndiritu SW, Kassie M, Shiferaw B. 2014. Are there systematic gender differences in the adoption of sustainable agricultural intensification practices? Evidence from Kenya. Food Policy, 49: 117-127. DOI: https://doi.org/10.1016/j.foodpol.2014.0 6.010 
Nigussie Z, Tsunekawa A, Haregeweyn N, Adgo E, Nohmi M, Tsubo M, Aklog D, Meshesha DT, Abele S. 2017. Factors influencing small-scale farme rs' adoption of sustainable land management technologies in north-western Ethiopia. Land Use Policy, 67: 57-64. DOI: https://doi.org/10.1016/j.landusepol.2017 .05 .024

Nkomoki W, Bavorová M, Banout J. 2018. Adoption of sustainable agricultural practices and food security threats: Effects of land tenure in Zambia. Land Use Policy, 78: 532-538. DOI: https://doi.org/10.1016/j.landusepol.2018 .07 .021

Obayelu AE, Okuneye PA, Shittu AM, Afolami CA, Dipeolu AO. 2016. Determinants and the perceived effects of adoption of sustainable improved food crop technologies by smallholder farmers along the value chain in Nigeria. Journal of Agriculture and Environment for International Development (JAEID), 110. DOI: https://doi.org/10.12895/jaeid.20161.436

Okpara UT, Stringer LC., Akhtar-Schuster M. 2019. Gender and land degradation neutrality: A cross-country analysis to support more equitable practices. Land Degradation \& Development, 30: 13681378.

DOI: https://doi.org/10.1002/ldr.3326

Paudel GS, Thapa GB. 2004. Impact of social, institutional and ecological factors on land management practices in mountain watersheds of Nepal. Applied geography, 24: 35-55. DOI: https://doi.org/10.1016/j.apgeog.2003.08 .011

Pender J. 2004. Development pathways for hillsides and highlands: some lessons from Central America and East Africa. Food Policy, 29: 339-367. DOI: https://doi.org/10.1016/j.foodpol.2004.0 7.005

Pender J, Gebremedhin B. 2007. Determinants of Agricultural and Land Management Practices and Impacts on Crop Production and Household Income in the Highlands of Tigray, Ethiopia. Journal of African
Economies, 17: 395-450. DOI: https://doi.org/10.1093/jae/ejm028

Quillérou E. 2012. Costs of land degradation and benefits of land restoration: a review of valuation methods and suggested frameworks for inclusion into policymaking. CAB Reviews: Perspectives in Agriculture, Veterinary Science, Nutrition and Natural Resources, 7. DOI: https://doi.org/10.1079/PAVSNNR2012 7060

Rahman SA, Sunderland T, Roshetko JM, Healey JR. 2017. Facilitating smallholder tree farming in fragmented tropical landscapes: Challenges and potentials for sustainable land management. Journal of Environmental Management, 198: 110121. DOI: https://doi.org/10.1016/j.jenvman.2017.0 4.047

Rasul G, Thapa GB. 2007. The Impact of Policy and Institutional Environment on Costs and Benefits of Sustainable Agricultural Land Uses: The Case of the Chittagong Hill Tracts, Bangladesh. Environmental Management, 40: 272283. DOI: https://doi.org/10.1007/s00267-0050083-8

Razzaghi Borkhani F, Mohammadi Y. 2019. Perceived outcomes of Good Agricultural Practices (GAPs) technologies adoption in citrus farms of Iran (reflection of environment-friendly technologies). Environmental Science and Pollution Research, 26: 6829-6838. DOI: https://doi.org/10.1007/s11356-01804083-0

Saïdou A, Tossou RC, Kossou D, Sambieni S, Richards P, Kuyper TW. 2007. Land tenure and sustainable soil fertility management in central Benin: towards the establishment of a cooperation space among stakeholders. International Journal of Agricultural Sustainability, 5: 195-212.

DOI: https://doi.org/10.1080/14735903.2007.9 684822

Salé A, Folefack DP, Obwoyere GO, Lenah Wati N, Lendzemo WV, Wakponou A. 2014. Changements climatiques et déterminants d"adoption de la fumure 
organique dans la région semi-aride de Kibwezi au Kenya. Int. J. Biol. Chem. Sci., 8(2): 680-694. DOI: http://dx.doi.org/10.4314/ijbcs.v8i2.24

Schmidt E, Chinowsky P, Robinson S, Strzepek K. 2017. Determinants and impact of sustainable land management (SLM) investments: A systems evaluation in the Blue Nile Basin, Ethiopia. Agricultural Economics, 48: 613-627. DOI: https://doi.org/10.1111/agec.12361

Shepherd KD, Soule MJ. 1998. Soil fertility management in west Kenya: dynamic simulation of productivity, profitability and sustainability at different resource endowment levels. Agriculture, Ecosystems \& Environment, 71: 131145. DOI: https://doi.org/10.1016/S01678809(98)00136-4

Shiferaw B. 1998. Resource degradation and adoption of land conservation technologies in the Ethiopian Highlands: A case study in Andit Tid, North Shewa. Agricultural Economics, 18: 233-247. DOI: https://doi.org/10.1016/S01695150(98)00036-X

Shiferaw BA, Okello J, Reddy, RV. 2009. Adoption and adaptation of natural resource management innovations in smallholder agriculture: reflections on key lessons and best practices. Environment, Development and Sustainability, 11: 601-619. DOI: https://doi.org/10.1007/s10668-0079132-1

Sibanda M, Dube T, Mubango T, Shoko C. 2016. The utility of earth observation technologies in understanding impacts of land reform in the eastern region of Zimbabwe. Journal of Land Use Science, 11: 384-400. DOI: https://doi.org/10.1080/1747423X.2015. 1130756

Siedenburg JR. 2005. Critical knowledge differences and adaptation to shifting resource constraints: Evidence from Shinyanga District, Tanzania. Geografisk Tidsskrift-Danish Journal of Geography, 105: 57-65. DOI: https://doi.org/10.1080/00167223.2005.1 0649526
Silas SR. 2014. Reducing impact of land degradation in Tanzania: do incentive market based mechanisms work for sustainable land management? Journal of Sustainable Development, 7: 1. DOI: 10.5539/jsd.v7n6p1

Singh RK, Singh A, Pandey CB. 2014. Agrobiodiversity in rice-wheat-based agroecosystems of eastern Uttar Pradesh, India: implications for conservation and sustainable management. International Journal of Sustainable Development \& World Ecology, 21: 46-59. DOI: https://doi.org/10.1080/13504509.2013.8 69272.

Smyth AJ, Dumanski J, Spendjian G, Swift MJ. 1993. FESLM: An international framework for evaluating sustainable land management. FAO Rome.

Stringer LC, Fleskens L, Reed MS, de Vente J, Zengin M. 2014. Participatory Evaluation of Monitoring and Modeling of Sustainable Land Management Technologies in Areas Prone to Land Degradation. Environmental Management, 54: 1022-1042. DOI: https://doi.org/10.1007/s00267-0130126-5

Teklewold H, Kassie M, Shiferaw B. 2013. Adoption of Multiple Sustainable Agricultural Practices in Rural Ethiopia: Adoption of Multiple Sustainable Agricultural Practices. Journal of Agricultural Economics, 64: 597-623. DOI: https://doi.org/10.1111/14779552.12011

Teklewold H, Kohlin G. 2011. Risk preferences as determinants of soil conservation decisions in Ethiopia. Journal of Soil and Water Conservation, 66: 87-96. DOI: https://doi.org/10.2489/jswc.66.2.87

Tesfaye A, Negatu W, Brouwer R, van der Zaag P. 2014. Understanding soil conservation decision of farmers in the Gedeb watershed, Ethiopia: adoption of soil conservation measures by smallholder farmers. Land Degradation \& Development, 25: 71-79. DOI: https://doi.org/10.1002/ldr.2187

Teshager Abeje M, Tsunekawa A, Adgo E, Haregeweyn N, Nigussie Z, Ayalew Z, 
Elias A, Molla D, Berihun D. 2019. Exploring Drivers of Livelihood Diversification and Its Effect on Adoption of Sustainable Land Management Practices in the Upper Blue Nile Basin, Ethiopia. Sustainability, 11: 2991. DOI: https://doi.org/10.3390/su11102991

Teshome A, de Graaff J, Ritsema C, Kassie M. 2014. Farmers' perceptions about the influence of land quality, land fragmentation and tenure systems on sustainable land management in the north western Ethiopian highlands. Land Degradation \& Development, 2014. DOI: 10.1002/ldr.2298

Tey YS, Li E, Bruwer J, Abdullah AM, Brindal M, Radam A, Ismail MM, Darham S. 2017. Factors influencing the adoption of sustainable agricultural practices in developing countries: a review. Environmental Engineering and Management Journal, 16: 337-349. DOI: https://doi.org/10.30638/eemj.2017.034

Thierfelder C, Mutenje M, Mujeyi A, Mupangwa W. 2015. Where is the limit? lessons learned from long-term conservation agriculture research in Zimuto Communal Area, Zimbabwe. Food Security, 7: 15-31. DOI: https://doi.org/10.1007/s12571-0140404-y

Tougiani A, Guero C, Rinaudo T. 2009. Community mobilisation for improved livelihoods through tree crop management in Niger. GeoJournal, 74: 377.

Vidogbéna F, Adégbidi A, Tossou R, AssogbaKomlan F, Martin T, Ngouajio M, Simon S, Parrot L, Garnett ST, Zander KK. 2016. Exploring factors that shape smallscale farmers' opinions on the adoption of eco-friendly nets for vegetable production. Environment, development and sustainability, 18: 1749-1770. DOI: http://dx.doi.org/10.1007/s10668-0159717-z.

Viswanath S, Nair PKR., Kaushik PK, Prakasam U. 2000. Acacia nilotica trees in rice fields: A traditional agroforestry system in central India. Agroforestry systems, $\quad$ 50: $\quad 157-177 . \quad$ DOI: 10.1023/A:1006486912126

Waswa F, Eggers H, Kutsch T. 2002. Beyond land titling for sustainable management of agricultural land: lessons from Ndome and Ghazi in Taita-Taveta, Kenya. Journal of Agriculture and Rural Development in the Tropics and Subtropics (JARTS), 103: 107-115.

Wollni M, Andersson C. 2014. Spatial patterns of organic agriculture adoption: Evidence from Honduras. Ecological Economics, 97: $120-128 . \quad$ DOI: https://doi.org/10.1016/j.ecolecon.2013.1 1.010

Wossen T, Berger T, Mequaninte T, Alamirew B. 2013. Social network effects on the adoption of sustainable natural resource management practices in Ethiopia. International Journal of Sustainable Development \& World Ecology, 20: 477483.

DOI: https://doi.org/10.1080/13504509.2013.8 56048

Yabi JA, Bachabi FX, Labiyi IA, Odé CA, Ayena RL. 2018. Déterminants socioéconomiques de l'eadoption des pratiques culturales de gestion de la fertilité des sols utilisés dans la commune de Ouaké au Nord- Ouest du Bénin. Int. J. Biol. Chem. Sci., 10(2): 779-792. DOI: DOI: http://dx.doi.org/10.4314/ijbcs.v10i2.27

Zeweld W, Van Huylenbroeck G, Tesfay G, Azadi H, Speelman S. 2018. Impacts of Socio-Psychological Factors on Actual Adoption of Sustainable Land Management Practices in Dryland and Water Stressed Areas. Sustainability, 10: 2963. DOI: https://doi.org/10.3390/su10092963

Zurayk R, el-Awar F, Hamadeh S, Talhouk S, Sayegh C, Chehab A.-G., al Shab K. 2001. Using indigenous knowledge in land use investigations: a participatory study in a semi-arid mountainous region of Lebanon. Agriculture, Ecosystems \& Environment 86: 247-262. DOI: https://doi.org/10.1016/S01678809(00)00287-5. 